\title{
Motion Deblurring of Infrared Images
}

\author{
B. Oswald-Tranta \\ Inst. for Automation, University of Leoben, Peter-Tunnerstr.27, A-8700 Leoben, Austria \\ beate.oswald@unileoben.ac.at
}

\begin{abstract}
:
Infrared images of an uncooled micro-bolometer can show significant blurring effects by recording a moving object. The electrical signal in the pixel of a micro-bolometer detector decays exponentially, hence the moving object is mapped to more pixels resulting in a blurred image. Not only the contrast is corrupted by the motion, but also the temperature of the object seems to be significantly less. In this paper it is shown how such images can be deblurred and the true temperature with a good approximation restored. As the detection mechanism of a micro-bolometer camera is different to CCD or CMOS cameras, therefore the point spread function (PSF) needed for the deblurring restoration is also different. It is shown, how the exponential coefficient of the PSF can be calculated if the motion speed and the camera resolution are known, otherwise how it can be estimated from the image itself. Experimental examples are presented for motion deblurring used to restore images with linear or with rotational motion.
\end{abstract}

Key words: motion deblurring, micro-bolometer, Wiener filter, image restauration, temperature measurement.

\section{Introduction}

The elimination of motion blurring in CMOS and CCD camera images is an extensively investigated topic [1]. Even in the last years many research works have been inspired e.g. by the problems of incidental shake in handheld cameras.

Assuming only one motion direction, the problem can be reduced to a simple image deconvolution. Is the blurring kernel, the pointspread-function (PSF) known, then this is called as a non-blind deconvolution. On the other hand, if the PSF is not known, it has to be estimated first from the image itself, which is called as blind deconvolution. Additionally techniques, as e.g. Wiener filter, have been developed for suppressing the noise, which could be strongly amplified by the deconvolution itself $[1,2]$.

Motion blurring can be also observed in images of infrared cameras. Cooled cameras with high sensitive photonic detectors have usually very short integration time, typically $1-1.5 \mathrm{~ms}$ for room temperature measurements. Therefore moving objects are exposed sharply and the blurring can be neglected. In contrast, the electrical signal in the pixel of a microbolometer camera decays with a time constant of $10-15 \mathrm{~ms}$, therefore a significant blurring effect can be observed by recording of moving objects.

The PSF of a motion blurring in a CMOS camera is mainly a linear function with a necessary length and direction [1,2]. In contrast, the signal of a micro-bolometer camera decays with an exponential function, therefore it requires a PSF with an exponential decay [3].

Furthermore, by the deblurring of a CMOS camera image the main goal is to obtain an image with high contrast. But by the restoring infrared images, it is also expected that the intensity, i.e. the temperature values of the objects should be also reconstructed.

In this paper it is investigated how in infrared images of a micro-bolometer camera the motion distortion can be deblurred. The first section summarizes equations and derivations published earlier $[3,4]$. The further sections show different experimental results, how this technique can be used, how non-blind and blind deblurring can be carried out. It is also investigated how well the temperature can be restored. 


\section{Deblurring of micro-bolometer images}

The blurred image is described generally by the equation:

$g=h \otimes f+n$

where $f$ is the true image recorded in perfect conditions and $h$ is the so-called point-spread function representing the distortion of the acquisition. The convolution of these two $(h \otimes f)$ results the distorted image. $n$ denotes the additional noise and $g$ the real image, recorded by the camera. The PSF of a microbolometer camera for a moving object is an exponential function $[3,4]$

$h=\frac{1}{\tau_{i m}} e^{-\frac{x}{\tau_{i m}}}$, for $0 \leq x$, otherwise 0

where the decay factor $\tau_{\text {im }}$ depends on the speed of the moving object $(v)$, on the resolution of the recorded image $(r)$ and on the exponential decay constant of the electrical signal of the micro-bolometer detector $\left(\tau_{\text {camera }}\right)$ which has a typical value of about $10 \mathrm{~ms}$ :

$\tau_{\text {im }}=\tau_{\text {camera }} v r$

It is well-known, that the Fourier transformation changes the convolution into a multiplication of the spectra. Therefore, if $G, H, F$ and $N$ denote the Fourier transformation of the functions $g, h$, $f$ and $n$, then Eq.(1) can be written as:

$G=H \cdot F+N$

In order to obtain an image $\widetilde{f}$ close to the true image, the inverse Fourier transformation of $\mathrm{G} / \mathrm{H}$ can be calculated:

$\widetilde{f}=\mathfrak{F}^{-1}\left(\frac{H \cdot F+N}{H}\right)=\mathfrak{F}^{-1}\left(F+\frac{N}{H}\right)$

If there were no noise, then $\tilde{f}=f$, that means the image could be perfectly restored. Due to the exponential PSF of the micro-bolometer camera the noise is strongly amplified, especially its high frequency components [3]. Therefore a kind of low-pass filter is necessary for the deblurring. A good possibility is to use $[2,3]$ a parametrized Wiener filter

$$
\widetilde{f}=\mathfrak{F}^{-1}\left(\frac{|H|^{2}}{|H|^{2}+k} \cdot\left(F+\frac{N}{H}\right)\right)
$$

where $k$ is a small non-negative number. It is to note that if $k=0$, then Eq.(6) is reduced to Eq.(5).

\section{Motion experiments with a small ball}

In the experiments to test motion deblurring a small conveyor belt is moving with a specified speed up to $1.8 \mathrm{~m} / \mathrm{s}$ in the field-of-view of the camera (see Fig.1). As comparison two cameras have been used, a micro-bolometer camera (left side) and a camera with cooled InSb detector (right side). As this second one has an integration time of $1.5 \mathrm{~ms}$, the motion deblurring in its images is negligible.

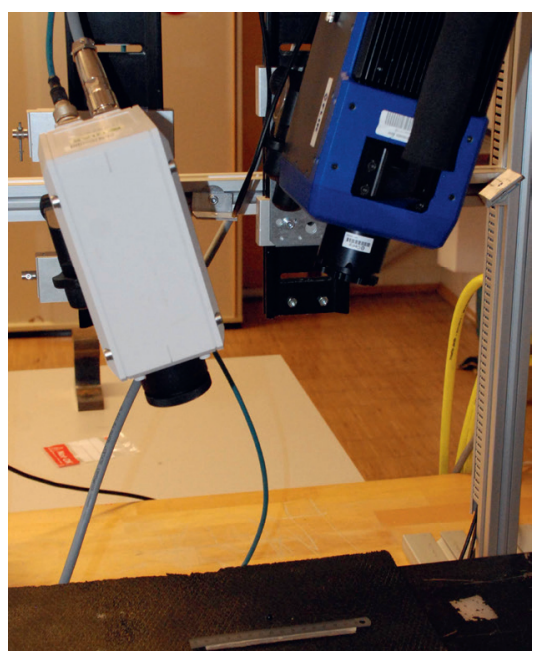

Fig.1. Experimental setup in the laboratory with a micro-bolometer and with a cooled photon detector, recording objects in motion on the conveyor belt.

A small warm ball has been moved with a $1 \mathrm{~m} / \mathrm{s}$ speed in the field-of-view of both cameras. Fig. 2 shows the image recorded by the microbolometer camera, which has been strongly blurred due to the motion. As the resolution of the image is $4.4 \mathrm{pixel} / \mathrm{mm}, \tau_{i m}$ is calculated by Eq.(3) as 44. In Fig. 3 three restored images are shown, deblurred with different $k$ values and in Fig.4 the temperature profiles for these three restored images are compared with the original, blurred image. If $k=0$, then the noise in the image is amplified, resulting a very noisy restored image. If $k=0.005$ the noise amplification is well suppressed due to the Wiener filtering and the deblurred image is sharp. If $k=0.05$, then this causes an elongation of the image.

The temperature of the ball was measured as $45^{\circ} \mathrm{C}$ with the cooled infrared camera. Due to the motion distortion the temperature seems to be only about $35^{\circ} \mathrm{C}$ with the micro-bolometer. But after restoring with $k=0.005$ the image shows also a maximal temperature of $45^{\circ} \mathrm{C}$. That means, not only the geometry but also the temperature value can be very well restored. In the distorted image the temperature is 'smeared' over more pixels, but with deblurring the same intensity is concentrated again to fewer pixels, and the measured temperature 
value is in very good agreement with the reality, measured with the cooled infrared camera.
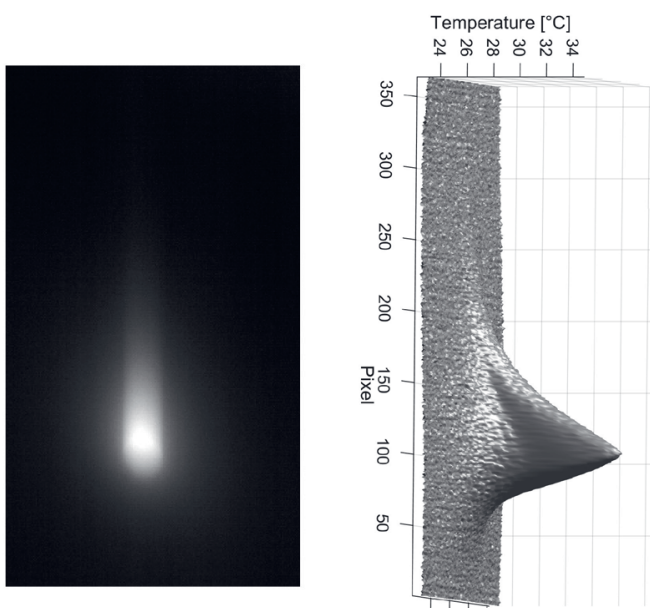

Fig.2. Infrared image of a ball, moving with $1 \mathrm{~m} / \mathrm{s}$; the image at the right shows the temperature as a surface and the exponential 'tail' can be well observed.
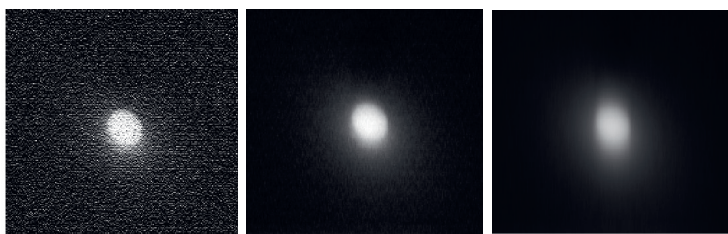

Fig.3. Images restored from Fig. 2 with different $k$ values: $0,0.005$ and 0.05 from the left to the right, respectively.

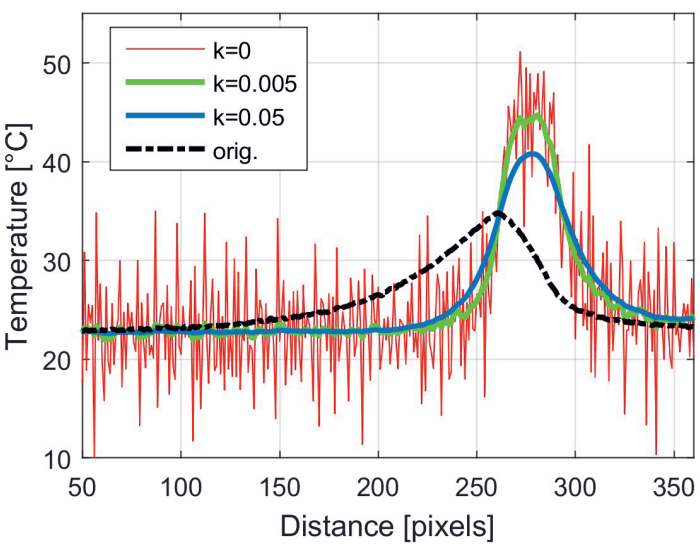

Fig.4. Temperature profiles through the three restored images of Fig. 3, compared with the original one in Fig. 2.

According to experiences [3] $k=0.005$ is a good compromise. If $k$ is too low, then the restored image is too noisy. On the other hand, if it is too high, then due to its low-pass filtering the edges of the object become smoothed, causing a blurring in the motion direction, which consequently results also in an apparently lower temperature.

The same image of Fig. 2 has been restored with different $\tau_{\text {im }}$ values (see Fig.5 and Fig.6). If $\tau_{\text {im }}$ is too low, then the image is not fully deblurred but a slight distortion remains in the restored image (see Fig.5 right and Fig.6) [3,4]. On the other hand, if $\tau_{\text {im }}$ is too high, then behind the object a kind of negative 'shadow' appears (see Fig.5 left and Fig.6). As the deblurring calculation keeps the total intensity of the whole image, therefore the restored temperature with a too low $\tau_{\text {im }}$ value is also too low, and with a too high one the restored temperature becomes also too high.
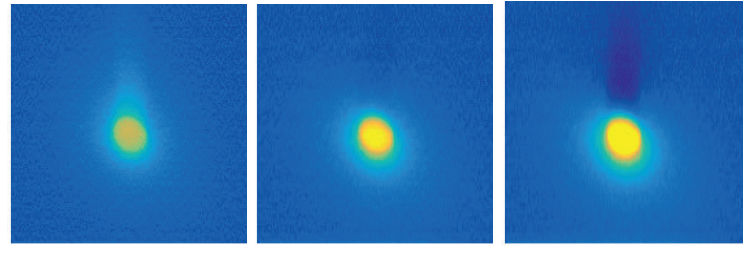

Fig.5. Images restored from Fig. 2 with different $\tau_{\text {im }}$ values: 22, 44 and 66 from the left to the right, respectively.

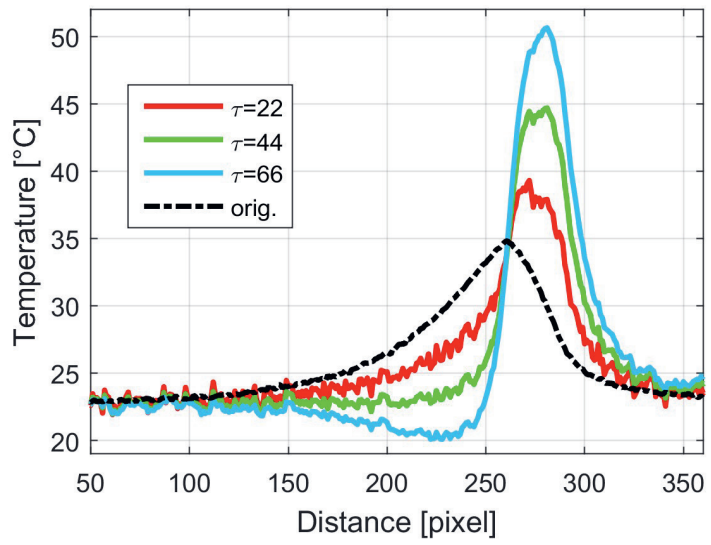

Fig.6. Temperature profiles through the three restored images of Fig.5, compared with the original one in Fig. 2.

It is to note, that the used $\tau_{\text {im }}$ values here are $50 \%$ and $150 \%$ of the correct value. The relative error of the restored temperature is approximately [3]

$$
\frac{\Delta T_{\text {restored }}-\Delta T_{\text {true }}}{\Delta T_{\text {true }}}=\frac{\tau_{2}-\tau_{\text {im }}}{\tau_{\text {im }}} \cdot \exp \left(-\frac{a}{\tau_{\text {im }}}\right)
$$

where

$\Delta T_{\text {restored }}=T_{\text {restored,object }}-T_{\text {restored, background }}$

$\Delta T_{\text {true }}=T_{\text {true,object }}-T_{\text {true,background }}$. a is the size of the object and $\tau_{2}$ is the used coefficient, instead of the correct $\tau_{i m}$. That means, the temperature difference between background and object is distorted due to the motion 
blurring and the larger the object, the smaller is this effect. For the example of Fig. 5 and Fig.6 calculated, as $a=30$ pixels, $\tau_{\text {im }}=44$, $\tau_{2}=0.5 \tau_{\text {im }}$, results in a temperature difference of about $0.25 \cdot \Delta T_{\text {true }} \approx 0.25 \cdot 22^{\circ} \mathrm{C} \approx 5.5^{\circ} \mathrm{C}$. If the used coefficient is closer to the real value, e.g. $\tau_{2}=0.9 \tau_{\text {im }}$, then the temperature difference would be about $1.1^{\circ} \mathrm{C}$. On the other side, it is also to note, that mainly small objects loose temperature due to incorrect deblurring, large object can be well restored.

\section{Sharpness of restored images}

In many cases, as e.g. thermographic nondestructive testing, non-calibrated cameras are used, as the temperature value itself is not important, only its distribution and a good contrast in the images [4-6].

Fig.7 demonstrates how well the sharpness of an image can be restored with the proposed deblurring algorithm. A $10 \mathrm{~cm} \times 10 \mathrm{~cm}$ metallic square ruler has been moved with the conveyor belt with $1 \mathrm{~m} / \mathrm{s}$ speed in the field of-view of the camera. Due to their different emissivity values the digits can be well read in a static image, but after motion distortion they cannot be recognized anymore. But after reconstruction the digits become readable again (see Fig.7 right side).

It is to note, that the object has a square shape, but the recorded image is squeezed. As the micro-bolometer camera is not a snapshot camera and it reads out the rows continuously, the bottom part of the object has been moved already further, bevor its values are read out in the detector. This is causing the squeezing of the object, which can be simply corrected by a projective transformation.
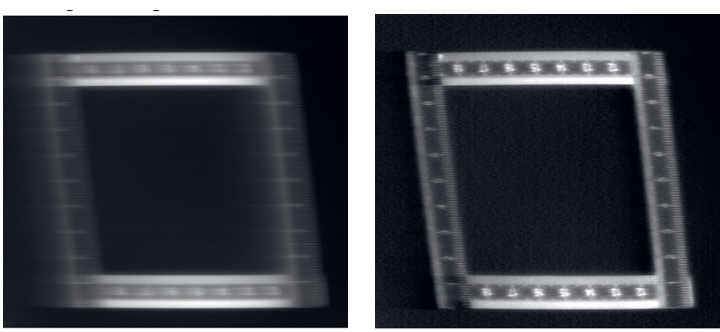

Fig.7. A metallic ruler during the motion (left image) and the deblurred image (right one)

Infrared cameras can be well used to detect failures, as e.g. surface cracks in objects. In the so-called active thermographic inspection first heat is introduced to the object in a specified way, and due to the distribution and the temporal change of the temperature the failures can be localized in the infrared images [4-6]. One of the heating techniques is to apply inductive heating, which is very efficient for ferro-magnetic materials, as e.g. for steel. Fig.8 shows a small bell, which is moved below an inductive coil, positioned on the left side, already outside of the image. The inductive heating causes a high temperature increase around a surface crack, which becomes very well visible and detectable in the deblurred image.
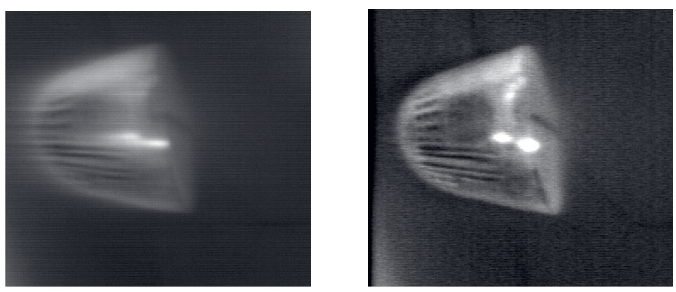

Fig.8. A small, inductively heated bell with a motion speed $1 \mathrm{~m} / \mathrm{s}$ (left image) and the deblurred image (right one)

\section{Blind deblurring}

In all the previous examples the exponential coefficient of the PSF and the moving direction has been determined once for the measurement setup and this has been used for restoring the images. This technique works in many cases, e.g. in process control or in nondestructive testing, when the objects always move with the same speed into the same direction.

But it is also possible to determine the PSF from the image itself, if there is somewhere a small hot point in the image. Fig. 2 shows the deblurred image of the moving ball. Specifying a profile, one can fit to this an exponential function (see Fig.9), which coefficient can be used as $\tau_{\text {im }}$.

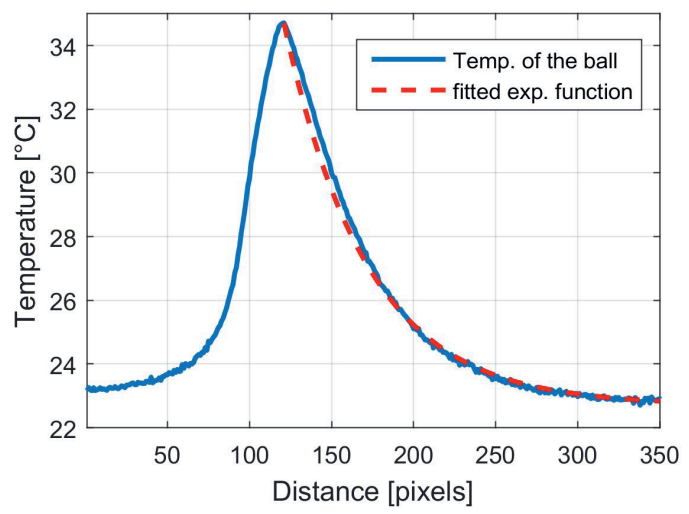

Fig.9. Blurred temperature profile from Fig. 2 and the fitted exponential function.

If the motion is linear, but its direction is not known, this can be also determined in many cases from the image itself [6]. Fig.10 demonstrates this situation: in first step the gradient image is calculated, in which the edges show the motion direction. After rotating the 
image with fitting the exponential function the full PSF can be determined. This technique works not only for such a simple case as a small ball in the image, but also for images where some points have significantly higher temperature than their surroundings[6].
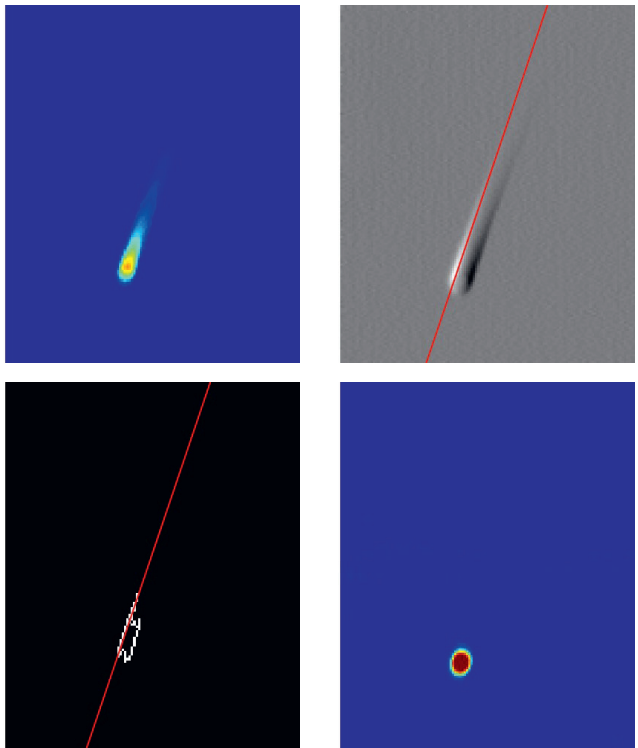

Fig.10.Infrared image of a small warm ball (top left), gradient image with marked motion direction (top right), edges of the gradient image (bottom left); restored image (bottom right).

This technique even works, if the motion is not linear, but its path is known. Using this a-priori knowledge, an additional step is necessary to transform the image first to a linear motion. E.g. a rotation can be transformed into a linear motion [4], which can be deblurred and then applying the inverse transformation an image with high contrast can be obtained.
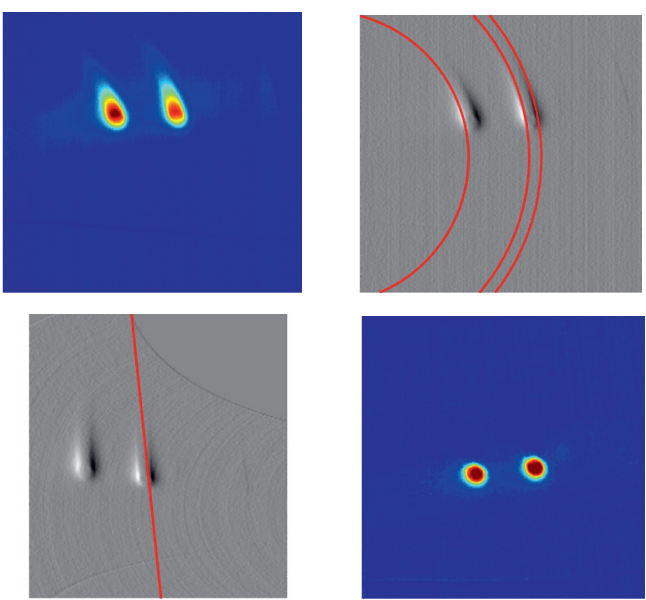

Fig.11.Infrared image of two small rotating balls (top left), gradient image to determine the rotation center (top right), image transformed to linear motion (bottom left); restored image (bottom right).

Fig.11 shows the infrared image of two small balls with the same temperature, fixed to a stick, which is rotating around one of its ends.
The ball at the right side has a larger radius and a larger speed. Due to the larger blurring its temperature seems to be lower. Fig.11 shows also the steps between for determining the rotation center and the transformed image to a linear motion. After the deblurring and the inverse transformation both balls have again a circular form and their temperature is also restored to the same value.

\section{Summary}

It has been shown that in many cases the motion blurring in the image of a microbolometer camera can be eliminated or strongly reduced and if the correct PSF is used, approximately the correct temperature can be also restored. If the speed and the direction of the motion are well known, then the PSF can be determined once, and used for the deblurring of the images. If the speed and the direction are not known, then the blurred image of a small hot point in the image corresponds to the pointspread-function, which can be then determined with a good approximation from the image itself.

\section{References}

[1] A.N.Rajagopalan, Rama Chellappa, Motion Deblurring: Algorithms and Systems, Cambridge Unversity Press, Cambridge, U.K. (2014)

[2] R.C.Gonzalez, R.E.Woods, S.L.Eddins, Digital Image Processing Using MATLAB, Gatesmark Publishing, (2009)

[3] B. Oswald-Tranta, M. Sorger, and P. O'Leary, "Motion deblurring of infrared images from a microbolometer camera," Journal of Infrared Phys. \& Technol., vol. 53, no. 4, pp. 274-279, (2010).

[4] B.Oswald-Tranta, "Automated thermographic non-destructive testing", Habilitation, University of Leoben, Austria, (2012).

[5] X. Maldague, Infrared and Thermal Testing, Nondestructive Testing Handbook, Vol. 3.ASNDT, (2001).

[6] B. Oswald-Tranta, M. Sorger, and P. O'Leary, "Thermographic crack detection and failure classification," J. Electron. Imaging, vol. 19(3), no. July-Sep, (2010).

[7] B. Oswald-Tranta and M. Sorger, "Scanning pulse phase thermography with line heating," QIRT Journal, vol. 9, no. 2, pp. 103-122, (2012). 